\title{
Improved GMM Estimation of AR(1) Time Series with a Root near 1
}

\author{
B. F. Chakalabbi ${ }^{1 *}$, Sanmati. Neregal ${ }^{2}$ and Sagar. Matur ${ }^{3}$ \\ ${ }^{1 *}$ Department of Statistics, Karnatak Arts College, Karnatak University, Dharwad - 580001, India \\ ${ }^{2}$ Department of Statistics, Karnatak Arts College, Karnatak University, Dharwad - 580001, India \\ ${ }^{3}$ Department of Statistics, Karnatak Arts College, Karnatak University, Dharwad - 580001, India \\ "Corresponding Author: bfckcd@gmail.com
}

Available online at: www.isroset.org

Received 16/Jan/2018, Revised 30/Jan/2018, Accepted 22/Feb/2018, Online 28/Feb/2018

\begin{abstract}
In this paper, to estimate AR(1) time series model First-difference GMM and Level GMM estimation methods have been considered, which have already performed well for estimation of AR(1) panel data model. A Monte Carlo simulation is carried out in order to study the performances of the above mentioned estimators and OLS estimator. Further, comparison among these estimators have been done in terms of bias and RMSE. Study reveals that, in many cases the OLS and First difference GMM estimators behave same in terms of Bias and RMSE. For all the negative values of autoregressive parameter the RMSE and bias of Level GMM estimator is larger than the remaining estimators. But in the case of positive values of autoregressive parameter Level GMM estimator performs better than First-difference GMM and OLS estimators especially, when sample size is small and autoregressive parameter is close to one.
\end{abstract}

Keywords - AR(1), First-difference GMM estimation, Level GMM estimation, OLS, Monte Carlo simulation

\section{INTRODUCTION}

There are numerous time series models available in the literature. The most widely used models are the Autoregressive (AR) models, the Integrated (I) models and the Moving Average (MA) models. A common approach for modelling time series is AR model. The first order autoregression $(\mathrm{AR}(1))$ is simple time series model, which can be analyzed through various standard methods. One of them is Ordinary Least Squares(OLS). The pioneers who worked in the area of OLS estimation of AR(1) time series model are [1] to [4] and more recent contributions include [5] to [7]. Their findings show that, the bias of the OLS estimator becomes large when sample size is small and an autoregressive parameter is near to unity.

The OLS method requires the assumption of orthogonality between the error term and regressor, which is often not satisfied in various applications. In such cases, the OLS estimator becomes inconsistent. To overcome this problem many estimation methods emerged in the study of estimation of AR(1) model. GMM being one of them relaxes the assumption of orthogonality and is used for estimation of AR(1) model, see [8] to [17]. Recent studies show an estimation and inference of a panel AR(1) model with small $\mathrm{T}$.

In the context of panel data, to estimate AR(1) model many estimation methods are proposed. Two consistent estimation methods among them are First-difference GMM (Dif) Proposed by [18] and Level GMM (Lev) introduced by [19]. In this paper, the above mentioned two estimation methods to estimate AR(1) time series model have been considered. In First-difference GMM method, the constant is removed from the AR(1) model and then instruments from the differenced AR(1) model are considered, where as in Level GMM estimation method the constant is removed directly from the instruments and GMM estimation is performed. The bias and RMSE of the above two estimators are compared along with OLS estimator through simulation results.

The paper is organized as follows; Section 2 provides model, assumptions and model estimators. Section 3 presents Monte Carlo simulation to investigate the performances of the considered estimators. Section 4 contains results and discussion. Finally, section 5 concludes the paper. 


\section{THE MODEL AND ESTIMATORS}

First order autoregressive time series model

$y_{t}=\alpha+\rho y_{t-1}+u_{t} \quad t=2,3, \ldots, T$.

(1) where

$\alpha$ is constant, $\rho$ is the parameter of interest with $|\rho|<1$.

Following are the assumptions:

\section{Assumption 1}

$\left\{u_{t}\right\}(t=2,3, \ldots, T)$ are i.i.d across time and independent of $y_{1}$ with $E\left(u_{t}\right)=0, \operatorname{Var}\left(u_{t}\right)=\sigma_{u}^{2}$.

\section{Assumption 2}

The autoregressive process $y_{t}$ is initialized at some random quantity $y_{1}$ with $E y_{1}^{2}<\infty$ allowing for stationarity by setting $y_{1}: N\left(0, \frac{\sigma^{2}}{1-\rho^{2}}\right)$

where, $y_{1}=\sum_{j=0}^{\infty} \rho^{j} u_{1-j}$

Based on the above assumptions two types of estimation methods are considered viz. First-difference GMM estimation method and Level GMM estimation method.

\section{First-Difference GMM Estimation:}

In the model (1), the constant $\alpha$ causes a correlation between the lagged endogeneous variable $y_{t-1}$ and error term $u_{t}$. First differences of model (1) is performed to remove the constant and one-step First-difference GMM estimator is proposed based on the following $(T-2)$ moment conditions

$$
E\left(Z_{d}^{\prime} \Delta u_{t}\right)=0
$$

where $Z_{d}$ is a $(T-2) \times(T-2)$ instrumental matrix employed by [20] and $\Delta u_{t}$ is a $(T-2) \times 1$ vector.

$$
Z_{d}=\left[\begin{array}{cccc}
y_{1} & 0 & \ldots & 0 \\
0 & y_{2} & \ldots & 0 \\
\vdots & \vdots & \ddots & \vdots \\
0 & 0 & \ldots & y_{T-2}
\end{array}\right] ; \quad \Delta u_{t}=\left[\begin{array}{c}
\Delta u_{3} \\
\Delta u_{4} \\
\vdots \\
\Delta u_{T}
\end{array}\right]
$$

Based on the moment conditions (2) the one-step firstdifference GMM estimator is obtained and is given by,

$$
\hat{\rho}_{d i f}=\left(\Delta y_{t-1}^{\prime} Z_{d} W_{d}^{-1} Z_{d}^{\prime} \Delta y_{t-1}\right)^{-1}\left(\Delta y_{t-1}^{\prime} Z_{d} W_{d}^{-1} Z_{d}^{\prime} \Delta y_{t}\right)
$$

where $\quad \Delta y_{t-1}^{\prime} \quad$ is the $1 \times(T-2) \quad$ vector $\left(\Delta y_{2}, \Delta y_{3}, \ldots, \Delta y_{T-1}\right), \Delta y_{t}^{\prime}$ is the $1 \times(T-2)$ vector $\left(\Delta y_{3}, \Delta y_{4}, \ldots, \Delta y_{T}\right), Z_{d}$ is a $(T-2) \times(T-2)$ matrix and $W_{d}=Z_{d}^{\prime} G_{d} Z_{d}$ is a $(T-2) \times(T-2)$ weight matrix with $G_{d}$ is same as $H$ in the estimator proposed by [18].

\section{Level GMM Estimation:}

On the basis of Arellano and Bover (1995) Level GMM estimator is proposed for $\operatorname{AR}(1)$ time series model. Here the constant $\alpha$ is wiped out from the instrumental variable. The one-step Level GMM estimator is based on the moment conditions

$$
E\left(Z_{l}^{\prime} u\right)=0
$$

where $Z_{l}$ is a $(T-2) \times(T-2)$ instrumental matrix employed by [20] and $u_{t}$ is a $(T-2) \times 1$ vector.

$Z_{l}=\left[\begin{array}{cccc}\Delta y_{2} & 0 & \ldots & 0 \\ 0 & \Delta y_{3} & \ldots & 0 \\ \vdots & \vdots & \ddots & \vdots \\ 0 & 0 & \ldots & \Delta y_{T-1}\end{array}\right] ; u_{t}=\left[\begin{array}{c}u_{3} \\ u_{4} \\ \vdots \\ u_{T}\end{array}\right]$

Based on the moment conditions (4) the one-step Level GMM estimator is obtained and is given by,

$\hat{\rho}_{l e v}=\left(y_{t-1}^{\prime} Z_{l} W_{l}^{-1} Z_{l}^{\prime} y_{t-1}\right)^{-1}\left(y_{t-1}^{\prime} Z_{l} W_{l}^{-1} Z_{l}^{\prime} y_{t}\right)$

where $y_{t-1}^{\prime}$ is the $1 \times(T-2)$ vector $\left(y_{2}, y_{3}, \ldots, y_{T-1}\right), y_{t}^{\prime}$ is the $1 \times(T-2)$ vector $\left(y_{3}, y_{4}, \ldots, y_{T}\right), Z_{l}$ is a $(T-2) \times(T-2) \quad$ matrix $\quad\left(Z_{l 1}, Z_{l 2}, \ldots, Z_{l N}\right) \quad$ and $W_{l}=Z_{l}^{\prime} G_{l} Z_{l}$, is a $(T-2) \times(T-2)$ weight matrix with $G_{l}$ is a $(T-2) \times(T-2)$ diagonal matrix.

\section{MONTE CARLO SIMULATION STUDY}

In this Monte Carlo simulation study, the data is generated from the following $\mathrm{AR}(1)$ model to investigate the finite sample performance of the above mentioned estimators. 


$$
y_{t}=\alpha+\rho y_{t-1}+u_{t}
$$

where $u_{t}$ is iid $N\left(0, \sigma^{2}\right)$. The initial condition is $y_{1}: N\left(0, \frac{\sigma^{2}}{1-\rho^{2}}\right)$ which is similar to the assumption in

[21]. For the parameters, $\alpha=1, \sigma^{2}=1$ and $\rho \in(-1,1)$ is considered, in particular $\rho \in[0.5,0.98]$ is considered. The sample size $T=5,10,20,40,50,100$ and 200 is chosen. The number of replications is 10000 .

\section{RESULTS AND DISCUSSION}

The results of the study are discussed through the tables and graphs respectively. When $\hat{\rho}$ (dif), $\hat{\rho}$ (lev) and $\hat{\rho}$ (ols) are compared, it is found that, in the case of $\mathrm{T}=5$ and 10 bias of $\hat{\rho}$ (lev) is the smallest and bias of $\hat{\rho}$ (ols) is the largest among mentioned estimators. $\hat{\rho}$ (dif) and $\hat{\rho}$ (ols) have almost equal bias for all the values of $\rho$ over the range $\mathrm{T}=20$ to 200. When $\mathrm{T}=20, \hat{\rho}$ (lev) has less bias than $\hat{\rho}$ (dif) and $\hat{\rho}$ (ols) except at the value of $\rho=0.5$. When the sample sizes are 40,50,100 and 200, $\hat{\rho}$ (lev) has lower bias than $\hat{\rho}$ (dif) and $\hat{\rho}$ (ols) except at the values of $\rho<0.7$, $\rho<0.75, \rho<0.8$ and $\rho<0.85$ respectively. In other words, when $\rho$ is near to unity, $\hat{\rho}$ (lev) is more preferable to the other two with regard to the bias.

Pertaining to the RMSE, for T $=5$, the RMSE of $\hat{\rho}$ (lev) is the smallest and bias of $\hat{\rho}$ (ols) is the largest among three estimators. For the cases of $T \geq 10, \hat{\rho}$ (dif) and $\hat{\rho}$ (ols) have almost identical RMSE for all considered $\rho$ values. When T=10 and 20, the RMSE of $\hat{\rho}$ (lev) is lesser than the RMSE of $\hat{\rho}$ (dif) and $\hat{\rho}$ (ols). When the sample sizes are 40, 50, 100 and 200, the RMSE of $\hat{\rho}$ (lev) is smaller than $\hat{\rho}$ (dif) and $\hat{\rho}$ (ols) except at the values of $\rho<0.65$, $\rho<0.75, \rho<0.85$ and $\rho<0.9$ respectively.

The graphs are plotted in figures 1-4 for the simulation results. Figure 1 depicts the comparison of means of $\hat{\rho}$ (dif), $\hat{\rho}$ (lev) and $\hat{\rho}$ (ols) with reference to true line over the entire range of $\rho$. From all graphs in figure 1, it is observed that, in terms of bias, $\hat{\rho}$ (dif) and $\hat{\rho}$ (ols) perform almost same in all the cases except at $\mathrm{T}=5$. When $\rho<0, \hat{\rho}$ (lev) has greater bias than other two estimators, but when $\rho$ is near to unity $\hat{\rho}$ (lev) has small bias than other two. To understand clearly, in figure 2 , the means of above three estimators have been plotted only for $\rho>0.5 . \hat{\rho}$ (lev) has small bias when $\mathrm{T}$ is not so large. As $\mathrm{T}$ increases, bias of $\hat{\rho}$ (lev) also increases, although $\hat{\rho}$ (lev) has smallest bias for $\rho>0.80$ for all values of $\mathrm{T}$.

Figure 3 shows the distinction of RMSE of $\hat{\rho}$ (lev), $\hat{\rho}$ (dif) and $\hat{\rho}$ (ols) for entire range of $\rho$. From figure 3 , it is noticed that $\hat{\rho}$ (dif) and $\hat{\rho}$ (ols) behave almost same in all the cases except at $\mathrm{T}=5$. RMSE of $\hat{\rho}$ (lev) is largest in negative range of $\rho$. Next, it starts decreasing from -0.5 and performs better than other two estimators as $\rho$ approaches unity. For more clarity, the RMSE of above considered estimators is plotted in figure 4 for $\rho>0.5$. From figure 4, it is observed that, $\hat{\rho}$ (lev) has less RMSE, especially when T is too small. As $\mathrm{T}$ increases $\hat{\rho}$ (dif) and $\hat{\rho}$ (ols) perform better than $\hat{\rho}$ (lev) but as $\rho$ approaches unity $\hat{\rho}$ (lev) performs excellent than remaining two estimators.

\section{CONCLUSION}

In this study, an estimation of $\mathrm{AR}(1)$ time series model is done by using First-difference GMM (Arellano and Bond (1991))and Level GMM (Arellano and Bover (1995)) estimation methods. Monte Carlo simulation is carried out to investigate the performances of the considered estimators. Based on the simulation results, it is observed that, for all the negative values of $\rho$ the RMSE and bias of Level GMM estimator is larger than the remaining estimators. In the case of $\rho<0$ in terms of bias and RMSE, Level GMM estimator does not perform best. But in the case of $\rho>0$, especially when $\rho$ is close to unity, the Level GMM estimator has small bias and is more efficient than First-difference GMM and OLS estimators. 
Table 1: Simulation results of comparison of the Bias and RMSE of $\hat{\rho}$ (dif), $\hat{\rho}$ (lev) and $\hat{\rho}$ (ols) (T=5,10,20 and 40).

\begin{tabular}{|c|c|c|c|c|c|c|c|c|c|c|c|c|}
\hline \multirow{2}{*}{$\mathrm{T}$} & & \multicolumn{11}{|c|}{$\rho$} \\
\hline & & 0.50 & 0.55 & 0.60 & 0.65 & 0.70 & 0.75 & 0.80 & 0.85 & 0.90 & 0.95 & 0.98 \\
\hline \multirow{9}{*}{5} & $\hat{\rho}($ dif $)$ & 0.018 & 0.023 & 0.051 & 0.053 & 0.084 & 0.114 & 0.121 & 0.139 & 0.174 & 0.208 & 0.232 \\
\hline & $\hat{\rho}(\mathrm{lev})$ & 0.593 & 0.592 & 0.61 & 0.623 & 0.648 & 0.674 & 0.7 & 0.718 & 0.749 & 0.783 & 0.798 \\
\hline & $\hat{\rho}$ (ols) & -0.034 & -0.03 & -0.01 & -0.009 & 0.01 & 0.029 & 0.039 & 0.052 & 0.071 & 0.095 & 0.112 \\
\hline & Bias(dif) & -0.482 & -0.527 & -0.549 & -0.597 & -0.616 & -0.636 & -0.679 & -0.711 & -0.726 & -0.742 & -0.748 \\
\hline & Bias(lev) & 0.093 & 0.042 & 0.01 & -0.027 & -0.052 & -0.076 & -0.1 & -0.132 & -0.151 & -0.167 & -0.182 \\
\hline & Bias(ols) & -0.534 & -0.58 & -0.61 & -0.659 & -0.69 & -0.721 & -0.761 & -0.798 & -0.829 & -0.855 & -0.868 \\
\hline & RMSE(dif) & 0.842 & 0.907 & 0.928 & 0.958 & 0.939 & 0.982 & 0.975 & 1.01 & 1.031 & 1.055 & 1.066 \\
\hline & RMSE(lev) & 0.622 & 0.6 & 0.68 & 0.563 & 0.586 & 0.573 & 0.575 & 0.572 & 0.587 & 0.594 & 0.596 \\
\hline & RMSE(ols) & 0.682 & 0.721 & 0.743 & 0.786 & 0.816 & 0.84 & 0.873 & 0.907 & 0.936 & 0.958 & 0.972 \\
\hline \multirow{9}{*}{10} & $\hat{\rho}($ dif $)$ & 0.24 & 0.277 & 0.3 & 0.338 & 0.362 & 0.391 & 0.419 & 0.448 & 0.469 & 0.509 & 0.529 \\
\hline & $\hat{\rho}(\mathrm{lev})$ & 0.618 & 0.642 & 0.647 & 0.67 & 0.687 & 0.711 & 0.736 & 0.761 & 0.788 & 0.827 & 0.852 \\
\hline & $\hat{\rho}$ (ols) & 0.232 & 0.268 & 0.29 & 0.327 & 0.35 & 0.378 & 0.403 & 0.432 & 0.451 & 0.486 & 0.503 \\
\hline & Bias(dif) & -0.26 & -0.273 & -0.3 & -0.312 & -0.338 & -0.359 & -0.381 & -0.402 & -0.431 & -0.441 & -0.451 \\
\hline & Bias(lev) & 0.118 & 0.092 & 0.047 & 0.02 & -0.013 & -0.039 & -0.064 & -0.089 & -0.112 & -0.123 & -0.128 \\
\hline & Bias(ols) & -0.268 & -0.282 & -0.31 & -0.323 & -0.35 & -0.372 & -0.397 & -0.418 & -0.449 & -0.464 & -0.477 \\
\hline & RMSE(dif) & 0.418 & 0.424 & 0.446 & 0.454 & 0.474 & 0.487 & 0.506 & 0.521 & 0.543 & 0.554 & 0.566 \\
\hline & RMSE(lev) & 0.304 & 0.293 & 0.287 & 0.282 & 0.277 & 0.279 & 0.278 & 0.277 & 0.286 & 0.289 & 0.289 \\
\hline & RMSE(ols) & 0.413 & 0.421 & 0.442 & 0.452 & 0.472 & 0.487 & 0.506 & 0.522 & 0.546 & 0.56 & 0.573 \\
\hline \multirow{9}{*}{20} & $\hat{\rho}($ dif $)$ & 0.372 & 0.411 & 0.455 & 0.489 & 0.53 & 0.568 & 0.607 & 0.638 & 0.672 & 0.707 & 0.733 \\
\hline & $\hat{\rho}(\mathrm{lev})$ & 0.668 & 0.68 & 0.701 & 0.716 & 0.736 & 0.757 & 0.781 & 0.808 & 0.842 & 0.88 & 0.904 \\
\hline & $\hat{\rho}$ (ols) & 0.371 & 0.41 & 0.454 & 0.488 & 0.528 & 0.566 & 0.605 & 0.635 & 0.669 & 0.703 & 0.728 \\
\hline & Bias(dif) & -0.128 & -0.139 & -0.145 & -0.161 & -0.17 & -0.182 & -0.193 & -0.212 & -0.228 & -0.243 & -0.247 \\
\hline & Bias(lev) & 0.168 & 0.13 & 0.101 & 0.066 & 0.036 & 0.007 & -0.019 & -0.042 & -0.058 & -0.07 & -0.076 \\
\hline & Bias(ols) & -0.129 & -0.14 & -0.146 & -0.162 & -0.172 & -0.184 & -0.195 & -0.215 & -0.231 & -0.247 & -0.252 \\
\hline & RMSE(dif) & 0.249 & 0.254 & 0.256 & 0.266 & 0.269 & 0.277 & 0.277 & 0.292 & 0.3 & 0.31 & 0.312 \\
\hline & RMSE(lev) & 0.242 & 0.217 & 0.199 & 0.187 & 0.172 & 0.168 & 0.166 & 0.164 & 0.162 & 0.16 & 0.159 \\
\hline & RMSE(ols) & 0.249 & 0.254 & 0.256 & 0.265 & 0.268 & 0.277 & 0.277 & 0.292 & 0.301 & 0.311 & 0.314 \\
\hline \multirow{9}{*}{40} & $\hat{\rho}($ dif $)$ & 0.434 & 0.481 & 0.526 & 0.57 & 0.616 & 0.661 & 0.704 & 0.748 & 0.786 & 0.827 & 0.849 \\
\hline & $\hat{\rho}(\mathrm{lev})$ & 0.69 & 0.709 & 0.726 & 0.748 & 0.768 & 0.791 & 0.813 & 0.844 & 0.873 & 0.915 & 0.939 \\
\hline & $\hat{\rho}$ (ols) & 0.435 & 0.482 & 0.527 & 0.57 & 0.616 & 0.662 & 0.705 & 0.749 & 0.787 & 0.828 & 0.849 \\
\hline & Bias(dif) & -0.066 & -0.069 & -0.074 & -0.08 & -0.084 & -0.089 & -0.096 & -0.102 & -0.114 & -0.123 & -0.131 \\
\hline & Bias(lev) & 0.19 & 0.159 & 0.126 & 0.098 & 0.068 & 0.041 & 0.013 & -0.006 & -0.027 & -0.035 & -0.041 \\
\hline & Bias(ols) & -0.065 & -0.068 & -0.073 & -0.08 & -0.084 & -0.088 & -0.095 & -0.101 & -0.113 & -0.122 & -0.131 \\
\hline & RMSE(dif) & 0.159 & 0.157 & 0.159 & 0.16 & 0.158 & 0.158 & 0.157 & 0.158 & 0.163 & 0.165 & 0.169 \\
\hline & RMSE(lev) & 0.22 & 0.195 & 0.168 & 0.146 & 0.128 & 0.113 & 0.105 & 0.096 & 0.095 & 0.087 & 0.087 \\
\hline & RMSE(ols) & 0.159 & 0.156 & 0.158 & 0.159 & 0.157 & 0.157 & 0.156 & 0.157 & 0.162 & 0.164 & 0.168 \\
\hline
\end{tabular}


Table 2: Simulation results of comparison of the Bias and RMSE of $\hat{\rho}$ (dif), $\hat{\rho}$ (lev) and $\hat{\rho}$ (ols) (T=50,100 and 200).

\begin{tabular}{|c|c|c|c|c|c|c|c|c|c|c|c|c|}
\hline \multirow{2}{*}{$\mathrm{T}$} & & \multicolumn{11}{|c|}{$\rho$} \\
\hline & & 0.5 & 0.55 & 0.6 & 0.65 & 0.7 & 0.75 & 0.8 & 0.85 & 0.9 & 0.95 & 0.98 \\
\hline \multirow{9}{*}{50} & $\hat{\rho}(\mathrm{dif})$ & 0.445 & 0.494 & 0.541 & 0.587 & 0.633 & 0.678 & 0.724 & 0.769 & 0.81 & 0.852 & 0.874 \\
\hline & $\hat{\rho}$ (lev) & 0.694 & 0.713 & 0.733 & 0.752 & 0.775 & 0.796 & 0.822 & 0.852 & 0.881 & 0.921 & 0.947 \\
\hline & $\hat{\rho}(\mathrm{ols})$ & 0.446 & 0.495 & 0.542 & 0.588 & 0.635 & 0.679 & 0.725 & 0.771 & 0.811 & 0.853 & 0.875 \\
\hline & Bias(dif) & -0.055 & -0.056 & -0.059 & -0.063 & -0.067 & -0.072 & -0.076 & -0.081 & -0.09 & -0.098 & -0.106 \\
\hline & Bias(lev) & 0.194 & 0.163 & 0.133 & 0.102 & 0.075 & 0.046 & 0.022 & 0.002 & -0.019 & -0.029 & -0.033 \\
\hline & Bias(ols) & -0.054 & -0.055 & -0.058 & -0.062 & -0.065 & -0.071 & -0.075 & -0.079 & -0.089 & -0.097 & -0.105 \\
\hline & RMSE(dif) & 0.139 & 0.14 & 0.136 & 0.137 & 0.134 & 0.133 & 0.131 & 0.13 & 0.135 & 0.134 & 0.138 \\
\hline & RMSE(lev) & 0.217 & 0.19 & 0.164 & 0.14 & 0.12 & 0.103 & 0.09 & 0.082 & 0.082 & 0.073 & 0.071 \\
\hline & RMSE(ols) & 0.139 & 0.139 & 0.135 & 0.136 & 0.133 & 0.132 & 0.13 & 0.129 & 0.133 & 0.132 & 0.136 \\
\hline \multirow{9}{*}{100} & $\hat{\rho}$ (dif) & 0.474 & 0.52 & 0.569 & 0.617 & 0.665 & 0.713 & 0.762 & 0.809 & 0.856 & 0.9 & 0.925 \\
\hline & $\hat{\rho}(\mathrm{lev})$ & 0.705 & 0.723 & 0.745 & 0.766 & 0.788 & 0.812 & 0.837 & 0.865 & 0.899 & 0.936 & 0.963 \\
\hline & $\hat{\rho}$ (ols) & 0.476 & 0.522 & 0.571 & 0.619 & 0.668 & 0.716 & 0.764 & 0.812 & 0.859 & 0.904 & 0.928 \\
\hline & Bias(dif) & -0.026 & -0.03 & -0.031 & -0.033 & -0.035 & -0.037 & -0.038 & -0.041 & -0.044 & -0.05 & -0.055 \\
\hline & Bias(lev) & 0.205 & 0.173 & 0.145 & 0.116 & 0.088 & 0.062 & 0.037 & 0.015 & -0.001 & -0.014 & -0.017 \\
\hline & Bias(ols) & -0.024 & -0.028 & -0.029 & -0.031 & -0.032 & -0.034 & -0.036 & -0.038 & -0.041 & -0.046 & -0.052 \\
\hline & RMSE(dif) & 0.093 & 0.092 & 0.09 & 0.088 & 0.085 & 0.082 & 0.079 & 0.076 & 0.074 & 0.073 & 0.074 \\
\hline & RMSE(lev) & 0.215 & 0.185 & 0.159 & 0.132 & 0.107 & 0.086 & 0.068 & 0.056 & 0.048 & 0.043 & 0.039 \\
\hline & RMSE(ols) & 0.092 & 0.091 & 0.089 & 0.087 & 0.083 & 0.08 & 0.077 & 0.074 & 0.071 & 0.069 & 0.07 \\
\hline \multirow{9}{*}{200} & $\hat{\rho}($ dif $)$ & 0.483 & 0.534 & 0.581 & 0.632 & 0.68 & 0.73 & 0.779 & 0.826 & 0.876 & 0.923 & 0.95 \\
\hline & $\hat{\rho}$ (lev) & 0.709 & 0.73 & 0.75 & 0.773 & 0.795 & 0.819 & 0.845 & 0.874 & 0.907 & 0.945 & 0.971 \\
\hline & $\hat{\rho}$ (ols) & 0.487 & 0.537 & 0.585 & 0.635 & 0.684 & 0.734 & 0.783 & 0.831 & 0.881 & 0.929 & 0.955 \\
\hline & Bias(dif) & -0.017 & -0.016 & -0.019 & -0.018 & -0.02 & -0.02 & -0.021 & -0.024 & -0.024 & -0.027 & -0.03 \\
\hline & Bias(lev) & 0.209 & 0.18 & 0.15 & 0.123 & 0.095 & 0.069 & 0.045 & 0.024 & 0.007 & -0.005 & -0.009 \\
\hline & Bias(ols) & -0.013 & -0.013 & -0.015 & -0.015 & -0.016 & -0.016 & -0.017 & -0.019 & -0.019 & -0.021 & -0.025 \\
\hline & RMSE(dif) & 0.064 & 0.063 & 0.061 & 0.058 & 0.057 & 0.054 & 0.051 & 0.049 & 0.045 & 0.042 & 0.041 \\
\hline & RMSE(lev) & 0.214 & 0.185 & 0.156 & 0.13 & 0.104 & 0.081 & 0.059 & 0.043 & 0.033 & 0.026 & 0.022 \\
\hline & RMSE(ols) & 0.063 & 0.062 & 0.060 & 0.057 & 0.055 & 0.052 & 0.049 & 0.045 & 0.041 & 0.037 & 0.036 \\
\hline
\end{tabular}

$\hat{\rho}($ dif $)=$ Difference GMM estimator, $\hat{\rho}(\mathrm{lev})=$ Level GMM estimator, $\hat{\rho}(\mathrm{ols})=$ Ordinary Least Square estimator, Bias(dif $)=$ Bias of Difference GMM estimator, Bias(lev)= Bias of Level GMM estimator, Bias(ols)= Bias of OLS estimator, RMSE= Root Mean Square Error, RMSE(dif)= RMSE of Difference GMM estimator, RMSE(lev)=RMSE of Level GMM estimator, RMSE(ols)=RMSE of OLS estiator 

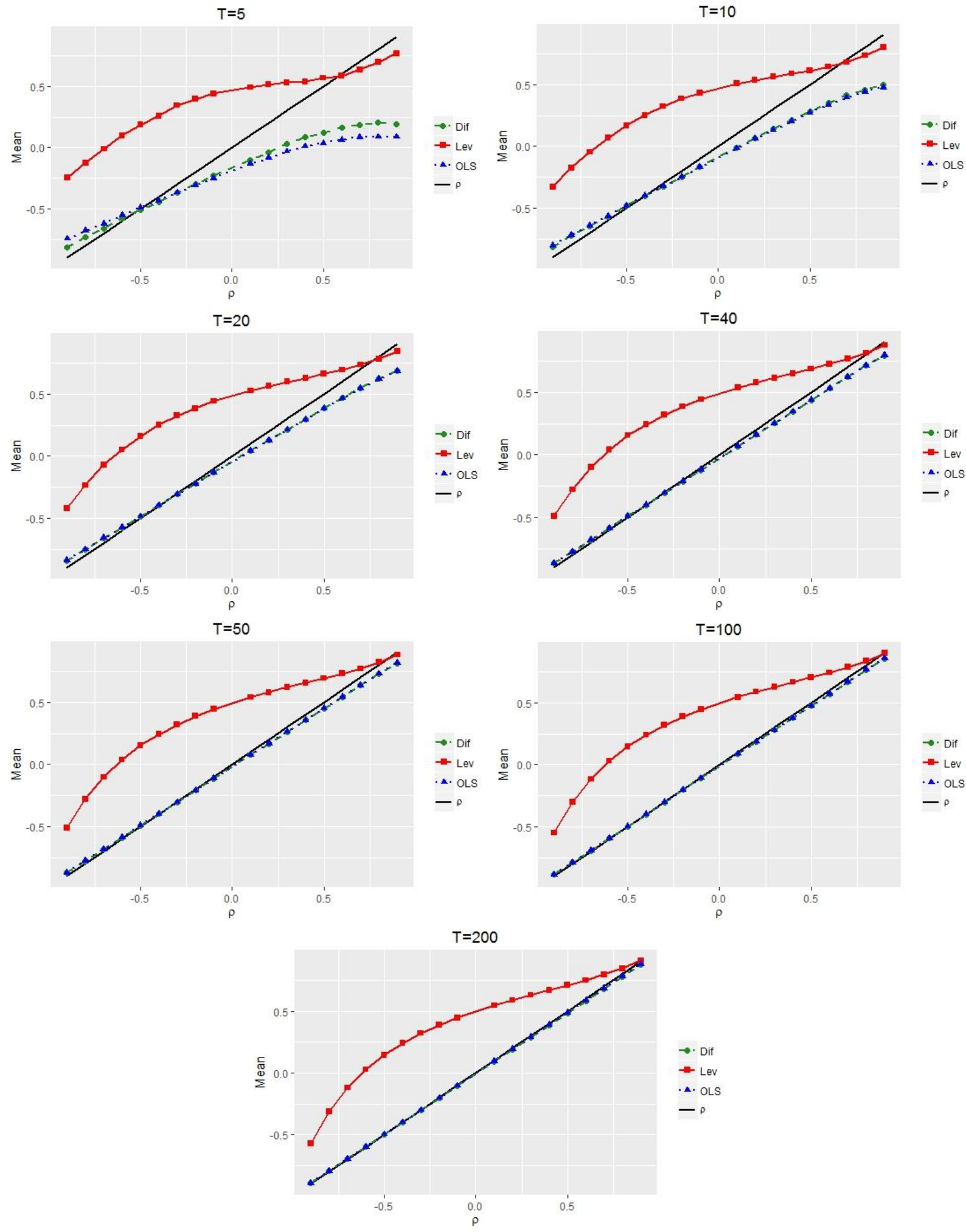

Figure 1: Means of Difference GMM, Level GMM and OLS estimators $(-1<\rho<1)$. 

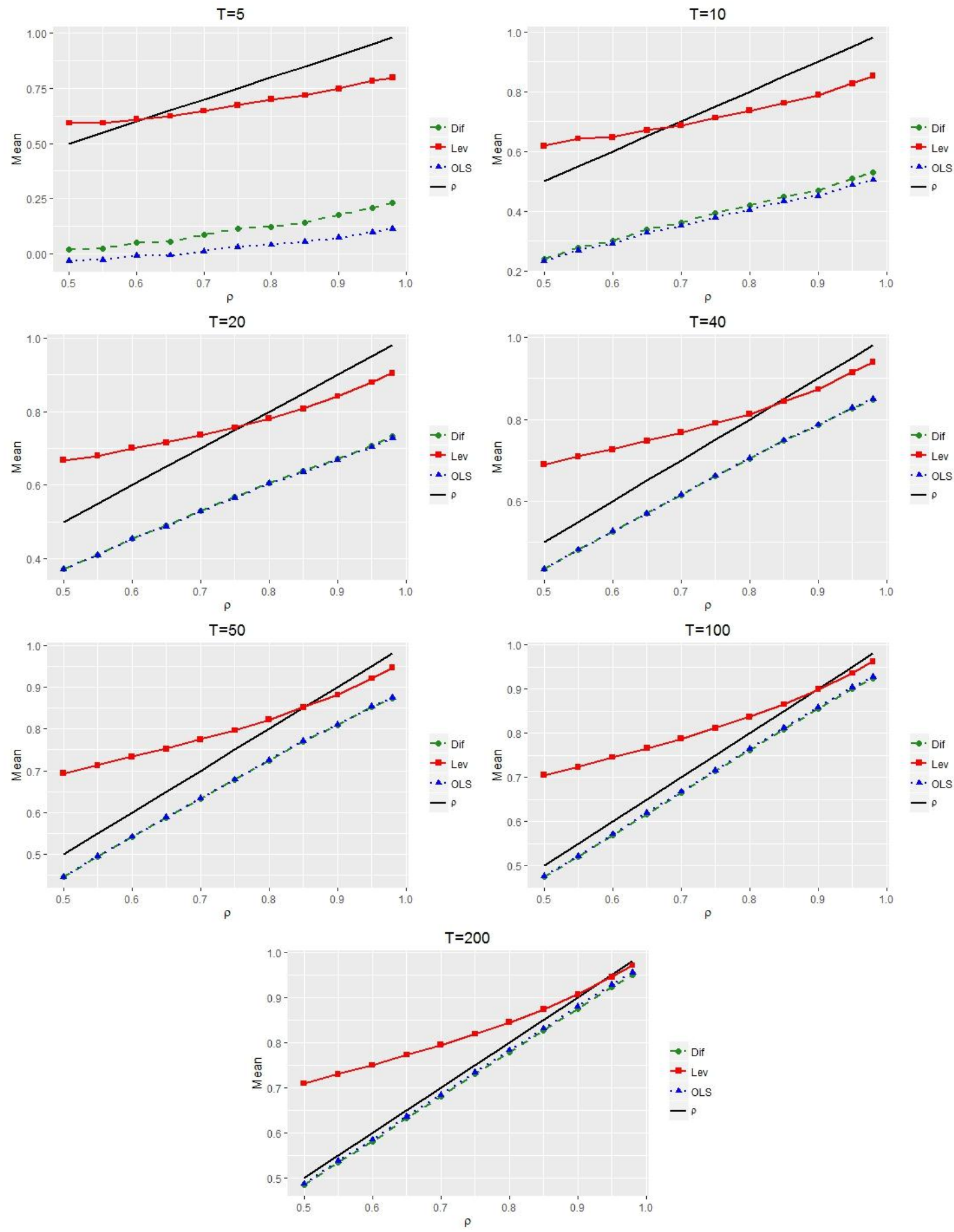

Figure 2: Means of Difference GMM, Level GMM and OLS estimators ( $\rho \geq 0.5)$. 

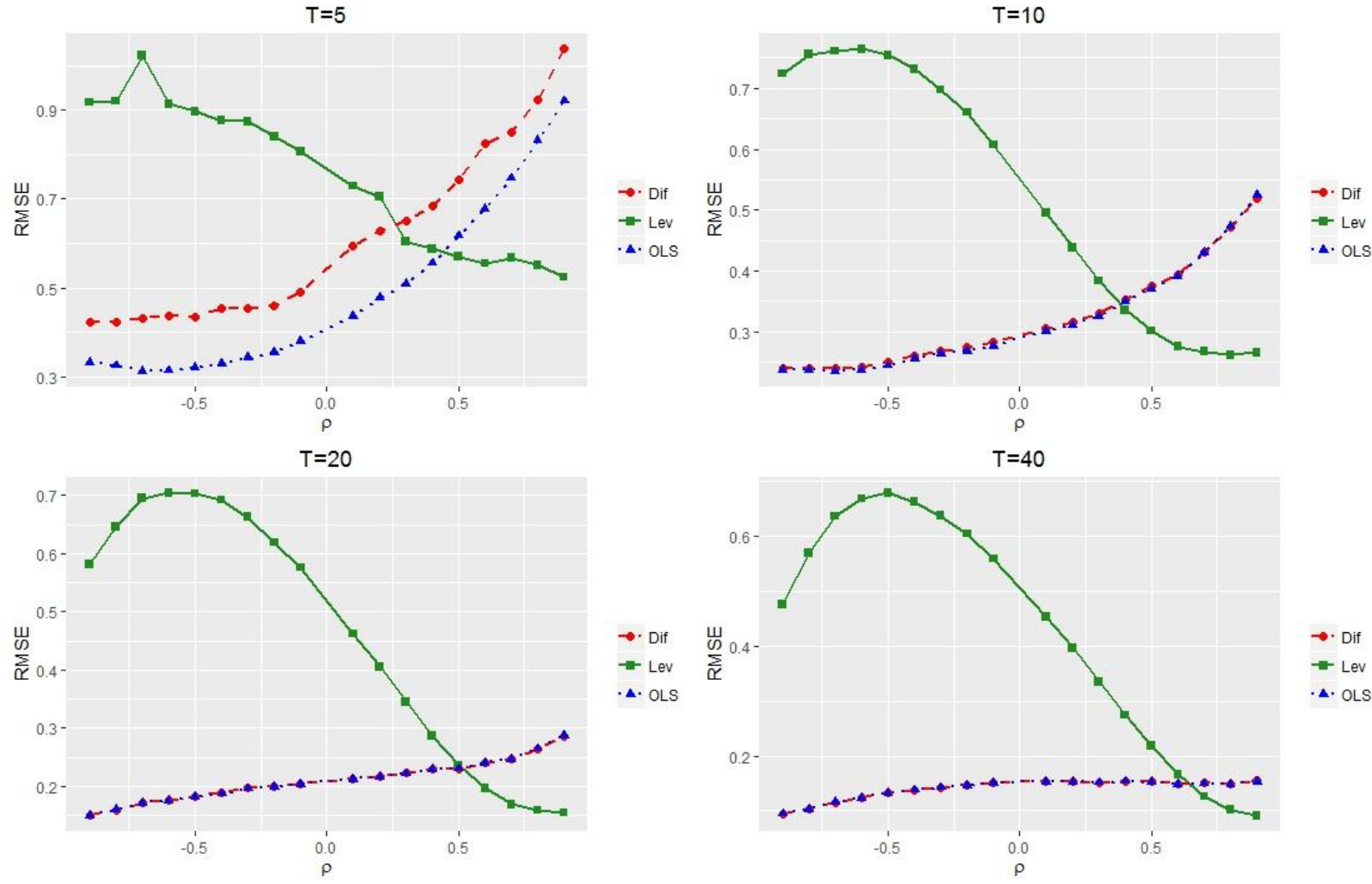

$\rightarrow$ Dif

$\rightarrow$ Lev

4. OLS
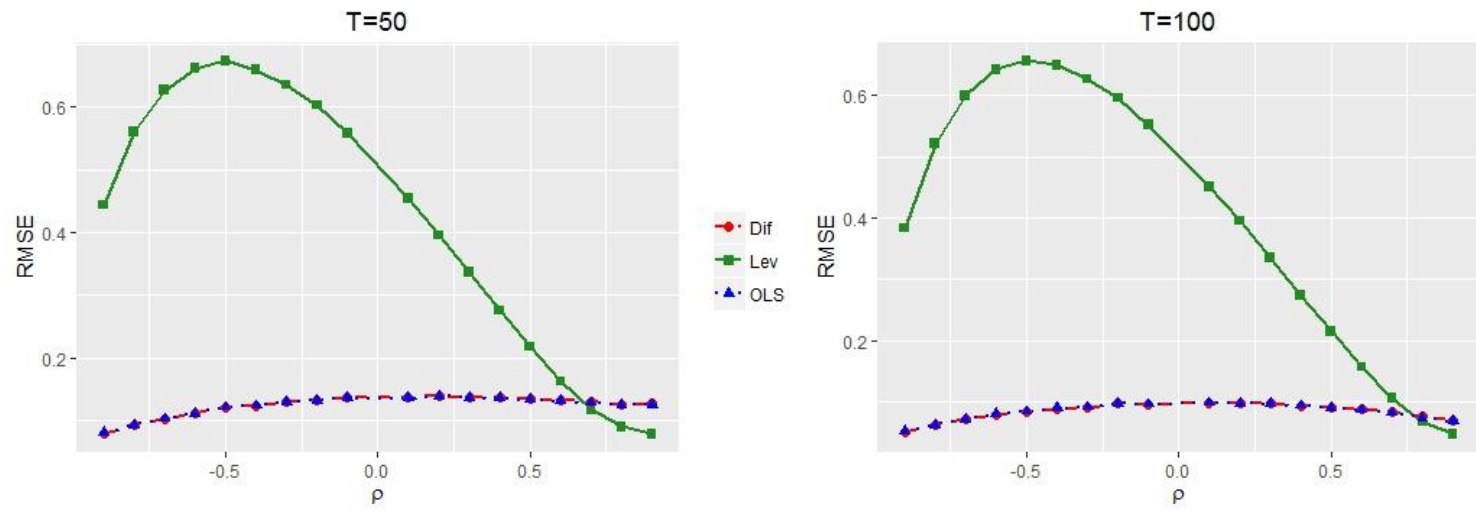

$\rightarrow$ Dif

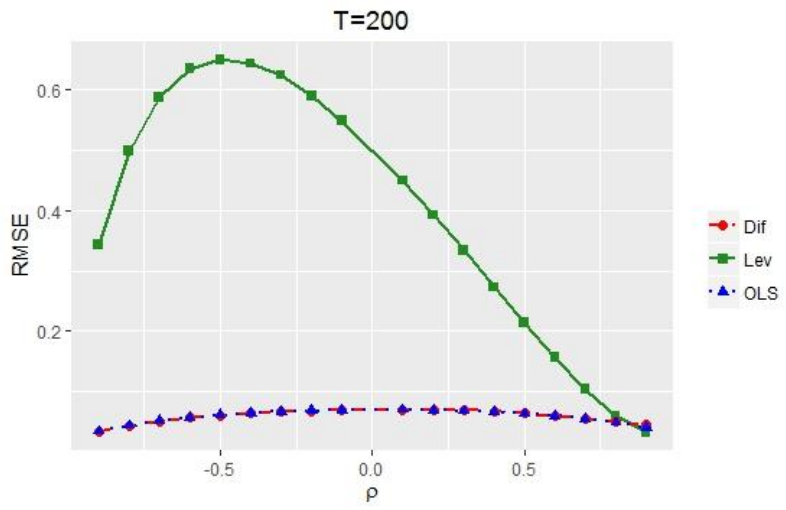

Figure 3: RMSEs of Difference GMM, Level GMM and OLS estimators $(-1<\rho<1)$. 

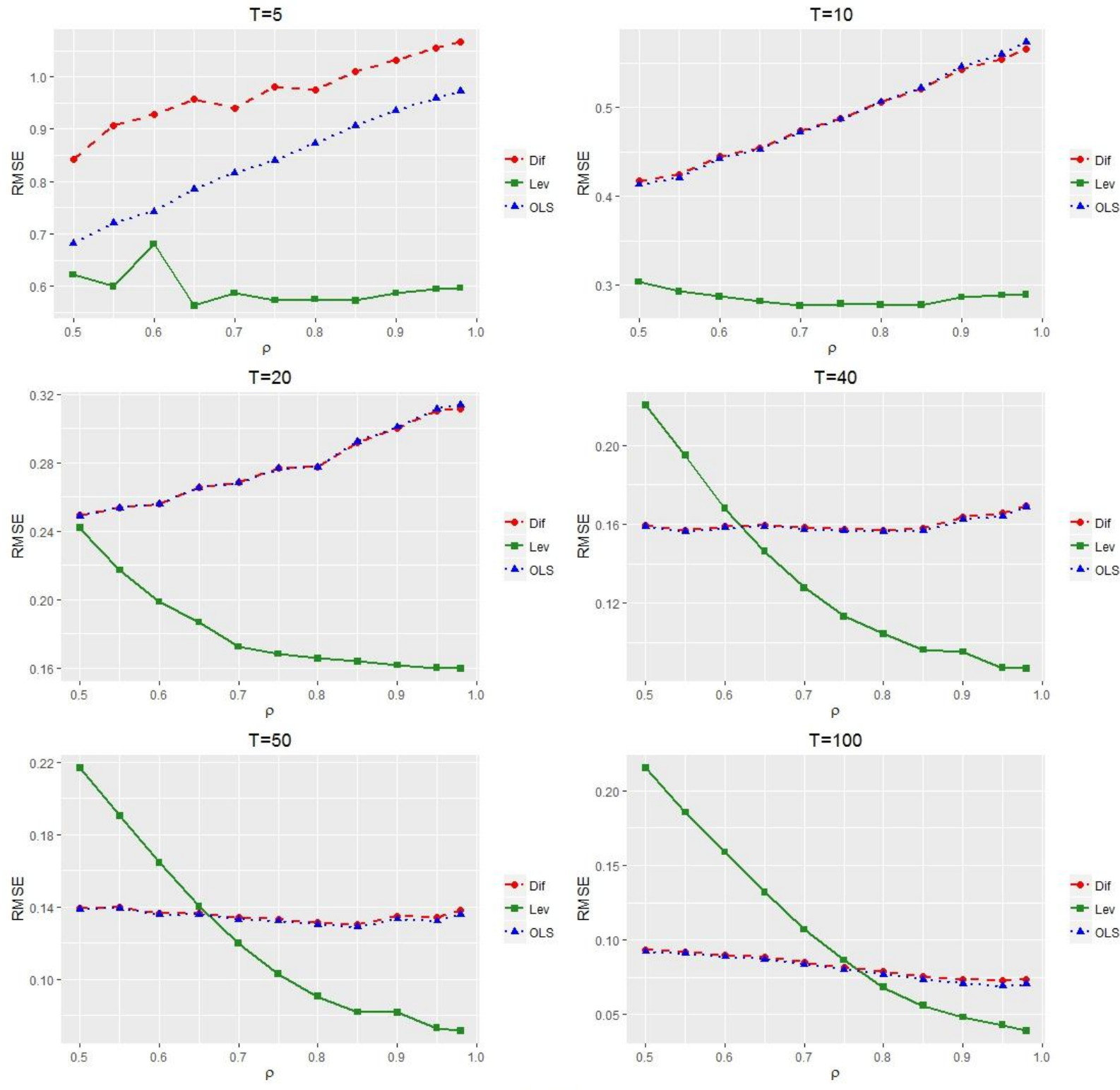

4. OLS

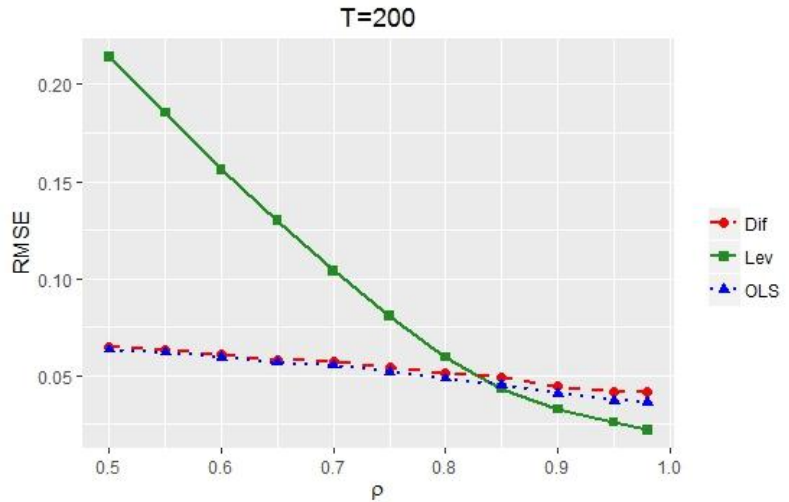

Figure 4: RMSEs of Difference GMM, Level GMM and OLS estimators ( $\rho \geq 0.5$ ). 


\section{REFERENCES}

[1] G.H. Orcutt, "A Study of the Autoregressive Nature of the Times Series Used for Tinbergenfs Model of the Economic System of the United States", Journal of the Royal Statistical Society, Series B, No. 1, pp. 1-45, 1948.

[2] L. Hurvicz, "Least Squares Bias in Time Series", In T. Koopmans (ed.), Statistical Inference in Dynamic Economic Models, Wiley, New York, pp. 365-383, 1950.

[3] F.H.C. Marriott, and J.A. Pope, "Bias in the Estimation of Autocorrelations", Biometrika, Vol. 41, No. (3/4), pp. 390-402, 1954.

[4] M.G. Kendall, "Note on the Bias in the Estimation of Autocorrelation", Biometrika, Vol. 41, No. (3-4), pp. 403-404, 1954.

[5] P.C.B. Phillips, "Approximations to Some Finite Sample Distributions Associated with a First Order Stochastic Difference Equation", Econometrica, Vol. 45, pp. 463-485, 1977.

[6] K. Tanaka, "Asymptotic expansions associated with the AR (1) model with unknown mean", Econometrica: Journal of the Econometric Society, No. 1, pp 1221-1231, 1983.

[7] P.Shaman, R. A. Stine, "The bias of autoregressive coefficient estimators", Journal of the American Statistical Association, Vol. 83, No. 403, pp. 842-848, 1988.

[8] L.P. Hansen, "Large Sample Properties of Generalized Method of Moments Estimators", Econometrica, Vol. 50, pp. 10291054, 1982.

[9] G. Chamberlain, "Asymptotic Efficiency in Estimation with Conditional Moments. Journal of Econometrics", Vol. 34, No. 3, pp. 305-334, 1987

[10] R. Cumby, J. Huinzinga, and M. Obstfeid, "Two-step, Two Stage Least Squares Estimation in models with Rational Expectations", journal of Econometrics, Vol. 21, No. 3, pp. 335-355, 1983.

[11] W. Newey, "Maximum Likelihood Specification Testing and Conditional Moment Tests", Econometrica, Vol. 53, pp. 10471070, 1985 a.

[12] W. Newey, "Generalized Method of Moments Specification Testing",Journal of Econometrics, Vol. 29, No. 3, pp. 229-256, $1985 \mathrm{~b}$.

[14] A. Pagan, and M. Wickens, "A Survey of Some Recent Econometric Methods", The Economic Journal, Vol. 99, No. 398, pp. 962-1025, 1989.
[15] W. H. Green, "Econoetric Analysis", Macmillan Publishing Company, New York, 1993.

[16] R. Davidson, and J. G. Mackinnon, "Estimation and Inference in Econometrics", Oxford University Press, New York, 1993.

[17] D.V. Vougas, "A comparison of LS/ML and GMM estimation in a simple AR(1) model", Communications in Statistics Simulation and Computation, Vol. 29, No. 1, pp. 239-258, 2000.

[18] M. Arellano, and S. Bond, "Some Tests of Specification for Panel Data: Monte Carlo Evidence and an Application to Employment Equations", Review of Economic Studies, Vol. 58, No. 2, pp. 277-297, 1991.

[19] M. Arellano, and O. Bover, "Another Look at the Instrumental Variable Estimation of Error-Components Models", Journal of Econometrics, Vol. 68, No. 1, pp. 29-51, 1995.

[20] M.J.G. Bun, and J.F. Kiviet, "The effects of dynamic feedbacks on LS and MM estimator accuracy in panel data models", Journal of Econometrics, Vol. 132, No. 2, pp. 409-444, 2006.

[21] C. Han, P. C. B. Phillips, and D. Sul, "Uniform Asymptotic Normality in Stationary and Unit Root Autoregression", Econometric Theory, Vol. 27, No. 6, pp. 1117-1151, 2011.

[13] W. Newey, and K. West, "Hypothesis Testing with Efficient Method of Moments Estimation", International Economic Review, Vol. 28, pp. 777-787, 1987.

\section{AUTHORS PROFILE}

Dr. B.F. Chakalabbi has completed his M.Sc, M.Phil and Ph.D. from Department of Statistics, Karnatak University, Dharwad. He is currently working as Associate Professor in Department of Statistics, Karnatak Arts college, Dharwad. His main research work focuses on Econometrics and Reliability.

Ms. Sanmati Neregal has comleted her M.Sc from Karnatak University, Dharwad, India in 2013. Currently she is pursuing $\mathrm{Ph} . \mathrm{D}$ in Time Series Econometrics from Karnatak University, Dharwad.

Mr. Sagar Matur has comleted his M.Sc from Karnatak University, Dharwad, India in 2013. Currently he is pursuing Ph.d in Panel Data Econometrics From Karnatak University, Dharwad. 